\title{
LncRNA LINC00665 promotes ovarian cancer cell proliferation and inhibits apoptosis via targeting miR-181a-5p/FHDC
}

\section{Suli Wang}

Maternity and Child Health Care Hospital

yingchun Wang

Maternity and Child Health Care Hospital

Jinhua Wang

Jiangsu Cancer Hospital \& Jiangsu Institute of Cancer Research

Jin Lu

Jiangsu Cancer Hospital \& Jiangsu Institute of Cancer Research

Ke Li ( $\nabla$ kelimdphd@tom.com )

The Affiliated Cancer Hospital of Nanjing Medical University

\section{Research}

Keywords: LINC00665, miR-181a-5p, FHDC1, human ovarian cancer

Posted Date: May 3rd, 2021

DOI: https://doi.org/10.21203/rs.3.rs-389237/v1

License: (c) (1) This work is licensed under a Creative Commons Attribution 4.0 International License. Read Full License 


\section{Abstract}

Previous reports indicate that LINC00665, a lincRNA, which is a naturally occurring long intergenic noncoding RNA exerts vital effect in a variety of cancers. Herein, we explore the role of LINC00665 in ovarian cancer. RT-qPCR was taken into the experiment to determine the expression level of LINC00665. Cell Counting Kit-8 (CCK-8), flow cytometry, wound healing, transwell, and EdU are employed to evaluate the effect of LINC00665 on cell proliferation, apoptosis, and migration in SKOV-3 and OVCAR-3 cells. The axis of LINC00665 and its specific miRNA, the miRNA target gene is explored in dual-luciferase reporter assay. Firstly, we find that LINC00665 is upregulated in ovarian cancer cell lines. Furthermore, not only the cell viability and in SKOV-3 and OVCAR-3 was reduced by LINC00665 knockdown but also cell proliferation and migration were inhibited. Afterwards, the bioinformatics analysis suggested that LNC00665 specifically binds to miR-181a-5p. Then, several studies confirmed that LINC00665 downregulated the miR-181a-5p in SKOV-3 and OVCAR-3 cells. The knockdown of miR-181a-5p evidently reverses the inhibitory effect of sh-LINC00662. Besides, FH2 Domain Containing 1 (FHDC1) has been proved to deed as an effective target of miR-181a-5p. In conclusion, the knockdown of LINC00665 facilitates ovarian cancer via development by sponging miR-181a-5p and upregulating FHDC1 expression.

\section{Introduction}

Among all the cancers in woman, ovarian cancer (OC) occurs a highly lethal malignancy with a high incidence of morbidity and mortality [1]. In United States, there are about 22 thousand new cases diagnosed and 14 thousand deaths related ovarian cancer in 2018 [2]. In China, the latest reported ovarian cancer deaths cases are 25 thousand [3]. Even therapy of 0 serve significant progresses in recent years, the advanced OC patients still has not improved median overall survival (OS) time [4]. Therefore, it was urgent to develop an effective strategy to improve early detection of ovarian cancer. However, despite increasing reports involves in the molecular level of ovarian cancer development, there is still rare about the exact mechanisms.

Long non-coding RNAs (IncRNAs) are the naturally occurring long intergenic non-coding RNA without coding proteins. The length of IncRNAs is over 200 base pairs, which differentiates them from the other non-coding RNAs [5]. During the past few decades, a great amount of reports have disclosed that IncRNAs took part in modulating gene expression through acting as scaffolds or sponges [6], which allows them to play some vital roles in cell proliferation, differentiation or apoptosis $[7,8]$. Numerous studies $[9,10]$ have provided clear evidence that the aberrant IncRNA plays an oncogenic or tumorsuppressive roles in ovarian cancer. The dysregulation of IncRNAs are expected to become a potential prognostic and diagnostic biomarker, as well as a therapeutic target for ovarian cancer, LINC00665 is first identified in oral premalignant lesions [11]. Next, LINC00665 is observed in several other tumors, including Hepatocellular Carcinoma (HCC) [12] and lung adenocarcinoma [13]. 
LINC00665 was reported to promote several kinds of human tumors progression, but its involvement in ovarian cancer remained unknown. According to our exploration, the important role of LINC00665, as an oncogene to promote ovarian cancer progression was clearly elucidated. First, we showed the function of LINC00665 in ovarian cancer cells. Then, a series of experiments were carried out to demonstrate that LINC00665 upregulated FHDC1 expression by sponging miR-181a-5p, indicating that LINC00665 might take part in the inactivation of the miR-181a-5p-FHDC1 pathway.

\section{Materials And Methods}

\section{Cell culture}

Human ovarian epithelial cell line (ISOE80) and human ovarian cancer cell lines (SKOV-3, A2780, OVCAR3 and H08910) were purchased from American Type Culture Collection (ATCC, Manassas, VA, USA). After resuscitation, ISOE80 was cultured in DMEM medium, while other cells were cultured in RPMI-1640 medium containing $10 \%$ fetal bovine serum (FBS, Beyotime, Beijing, China) in a humidified environment. When cells attained $70 \%$ to $80 \%$ confluences, transfection experiments could be conducted.

\section{Cell transfection}

LINC00665 shRNA constructs were generated by inserting the LINC00665-targeting shRNA sequences into pENTR/U6 plasmids. The NC mimic and miR-181a-5p mimic were provided by Ribobio corporation (Guangzhou, China). After $6 \mathrm{~h}$, fresh culture medium was added to the CFs with a confluence rate at $70 \%$. After 48-h post-transfection, plasmid transfection efficiency was measured by qRT-PCR.

\section{CCK8 assay}

After 48-h transfection, SKOV-3 and OVCAR-3 were treated with electroporation, and then were inoculated into 96 -well plates at $5 \times 10^{3}$ per well. After incubated for 0 hour, 24 hours, 48 hours and 72 hours, $10 \mu \mathrm{L}$ CCK8 solution were added and continuously incubated for 2 hours in the dark.

\section{EdU assay}

EdU assay was taken as described [14]. Briefly, after electroporation treatment, we seeded $5 \times 10^{3}$ SKOV-3 and OVCAR-3 into each well of the 96-well plates and cultured the cells for another 72 hours. Then, we incubated the cells with $10 \mu \mathrm{M}$ EdU for 4 hours, and stained DAPI. Finally, we analyzed the EdU-positive cells by fluorescence microscope (Leica, Hilden, Germany), 200x, and densitometric analyzed the percentage using ImageJ (Bethesda, MD, USA).

\section{Cell cycle and cell apoptosis}

After electroporation for transfection, we seeded the SKOV-3 and OVCAR-3 cells into 6 -well plates at $5 \times$ $10^{5}$ per well and cultured the cells for next 72 hours. Then, we harvested the tumor cells. For cell cycle, the cells were first incubated in ice-cold ethanol for 2 hours and then treated with RNase $A(0.2 \mathrm{mg} / \mathrm{mL}$, 
Sigma-Aldrich). Next, the cells were incubated with propidium iodide $(2 \mu \mathrm{L})$ at room temperature for $40 \mathrm{~min}$, and finally cell cycle detection was analyzed. For apoptosis, the harvested cells were treated with $\mathrm{PI}(10 \mu \mathrm{g} / \mathrm{mL})$ and V-fluorescein isothiocyanate $(10 \mu \mathrm{g} / \mathrm{mL})$, respectively.

\section{Wound healing assay}

Briefly, $5 \times 10^{5}$ SKOV-3 or OVCAR-3 were seeded into 6-well plate. When the cells were cultured to $90 \%$ confluence, a wound was made by a $100 \mu \mathrm{L}$ size pipette tip. After another 48 hours, the wound recovery area was evaluated under a light microscope.

\section{Transwell assay}

To detect the capacity of migration, the Transwell assay was performed [15]. After 48-h cell transfection, SKOV-3 or OVCAR-3 were firstly adjusted to the appropriate concentration. Subsequently, the upper chamber was added with adjusted cells, whereas the lower chamber was replaced by a medium supplement $15 \%$ FBS. After $24 \mathrm{~h}, 4 \%$ paraformaldehyde was used for fixation for 10 min and $0.1 \%$ crystal violet for staining for $30 \mathrm{~min}$, respectively. Five fields were randomly selected under an inverted microscope to represent the migration and invasion ability of cells in each group.

\section{Luciferase reporter assay}

The luciferase reporter was constructed by inserting the cDNA fragments containing the putative miR181a-5p binding site from LINC00665 or FHDC1 3'-UTR into pmirGLO Dual-Luciferase miRNA Target Expression Vectors (Promega, Madison, WI, USA). PmirGLO/LINC00665 or pmirGLO/FHDC1 3'-UTR constructs along with miR-181a-5p mimics were co-transfected into SKOV-3 and OVCAR-3 cells. Finally, the Dual-Luciferase Reporters' luciferase were measured activity according to the manufacturer's protocol.

\section{Immunofluorescence staining}

After 48-h transfection, SKOV-3 and OVCAR-3 were fixed in 4\% paraformaldehyde and permeabilized with xylene. Blocked with 5\% BSA in PBS, the cells were immunolabeled with primary antibody: FHDC (1:100; Proteintech) and then incubated with FITC-conjugated secondary antibody after washing. The nuclei were counterstained using DAPI (Invitrogen) and the cells were observed under a fluorescence microscope (Olympus, Japan).

\section{RT-qPCR analysis}

TRIpure reagent (Invitrogen, USA) was used to isolate the total RNA from samples and PrimeScript RT kit (TaKaRa, Otsu, Japan) was used for reverse transcription. After the sample was prepared, the expression level was detected with SYBR green, and GAPDH was controlled as internal parameter. $2^{-\Delta \Delta C t}$ methods represented the fold changes of gene expression and the experiments were conducted three times. Primers of LINC00665, miR-181a-5p, U6, FHDC1 and GAPDH were as described in previous studies [12, 
$16,17]$. LINC00665 sense, 5'AGCACCCCTAGTGTCAGTCA-3' and antisense, 5'-

TGGTCTCTAGGGAGGCAGAA-3'; miR-181a-5p sense, 5'-AACATTCAACGCTGTCGGTGAGT-3' and antisense, 5'-GTGCAGGGTCCGAGGT-3'; U6 sense, 5'-CTCGCTTCGGCAGCACA-3' and antisense, 5'AACGCTTCACGAATTTGCGT-3'; FHDC1 sense, 5'- ACATCCAGCGGGATGGTGAACT-3' and antisense, 5'GGAGCTCTTGTTTCCAGCATTCC-3'.

\section{Western blot}

According to the manufacture's instruction, the proteins were extracted and its concentration was measured. Subsequently, the prepared protein was separated by polyacrylamide-SDS gels and then transferred onto PVDF membranes (Roche, Switzerland). After blocking, the PVDF membrane was subjected to incubation with primary antibodies: FHDC1 (1:1000; cat. no. NBP1-93579; Novus Biologicals, Littleton, USA), MMP2 (1:1000; cat. no. 40994S; Cell Signaling Technology, Danvers, MA, USA), MMP9 (1:1000; cat. no. 13667S; Cell Signaling Technology), Cyclin D1 (1:1000; cat. no. 55506S; Cell Signaling Technology), p21 (1:1000; cat. no. 2947S; Cell Signaling Technology), Bcl-2 (1:1000; cat. no. ab185002; Abcam), Bax (1:500; cat. no. ab53154; Abcam), Cleaved Caspase-3 (1:500; cat. no. ab49822; Abcam), Cleaved Caspase-9 (1:1000; cat. no. 20750S; Cell Signaling Technology) or GAPDH (1:1000; cat. no. 5174S; Cell Signaling Technology). On the following day, the membrane were incubated with the secondary antibody at $37^{\circ} \mathrm{C}$ for $45 \mathrm{~min}$ and the intensity of protein expression was detected by ECL chemiluminescence (Beyotime, Beijing, China).

\section{Statistical Analysis}

GraphPad Prism (Version 6.01 for Windows) statistical software was used to perform statistical analysis. Student $t$ tests were employed to identify the significant differences between groups. Statistical significance difference was set at $p<0.05$.

\section{Results}

\section{LINC00665 is up-regulated in ovarian cancer cell lines}

To confirm whether LINC00665 took part in ovarian cancer, we employed RT-qPCR to compare its expression level in different OC cells (SKOV-3, A2780, OVCAR-3 and H08910) and human ovarian epithelial cell (ISOE80). The results demonstrated that LINC00665 level was significantly up-regulated in SKOV-3, A2780, OVCAR-3 and H08910 cell lines whereas decreased in ISOE80 (Fig. 1A). As the expression level of LINC00665 in SKOV-3 and OVCAR-3 were highest among above four cancer cells, we chose the two as cell models to explore biological function of LINC00665 in vitro.

\section{Knockdown of LINC00665 inhibits ovarian cancer cell proliferation and promotes the apoptosis}

In order to take the further exploration about the important role of LINC00665 in ovarian cancer, we transfected sh-RNA into SKOV-3 and OVCAR-3 cells which targets to LINC00665 (sh-LINC00665). The expression of LINC00665 was detected by RT-qPCR and the result indicated that LINC00665 is 
significantly down-regulated (Fig. 1B, p<0.01). CCK-8 assay depicted that sh-LINC00665 remarkably reduced the viability of SKOV-3 and OVCAR-3 cells (Fig. 1C, $p<0.01$ ). While EdU assay depicted that shLINC00665 remarkably reduced the EdU positive cells compared to sh-NC in both SKOV-3 and OVCAR-3 cells (Fig. 1D, $p<0.01$ ). Next, the data from flow cytometry assay indicated that sh-LINC00665 prominently increased the cells of ( $p<0.01$ ), while decreased $\mathrm{S}$ and G2/M phase cells (Fig. $1 \mathrm{E}, p<0.05$ ).

Meanwhile, the result of western blot showed that sh-LINC00665 remarkably reduced the expression of Cyclin D1 and increased the expression of p21 (Fig. 1F, $p<0.01$ ). Besides that, flow cytometry data delineated that silencing of LINC00665 can sharply induce apoptosis of OC cells (Fig. 1G, $p<0.01$ ). In Fig. $1 \mathrm{H}, \mathrm{Bax}$, Cleaved Caspase-3 and Cleaved Caspase-9 protein levels were up-regulated after treatment with sh-LINC00665, while Bcl-2 was down-regulated (Fig. $1 \mathrm{H}, p<0.01)$.

\section{Knockdown of LINC00665 inhibits ovarian cancer cell migration and invasion}

As presented in Fig. 2A, compared with the control group, the expression of LINC00665 reduced and the migratory ability of SKOV-3 and OVCAR-3 were remarkably repressed $(p<0.01)$. Besides, transwell assay delineated that sh-LINC00665 markedly inhibited the invasion of OC cells compared to sh-NC (Fig. 2B, $p<0.01)$. Additionally, western blot was explored to measure the cell migration related proteins. The result showed that sh-LINC00665 remarkably downregulated the transcription of MMP-2 and MMP-9 in SKOV-3 and OVCAR-3 cells compared to sh-NC (Fig. 2C, $p<0.01$ ).

\section{MiR-181a-5p is the target gene of LINC00665}

Since one way IncRNAs regulated gene expression was to act as a miRNA sponge, we predicted the potential miRNAs targets of LINC00665 in DIANA-LncBase (https://bigd.big.ac.cn). According to the research, miR-181a-5p was one of the potential targets (Fig. 3A). Previous, miR-181a-5p was reported to be significantly related with the overall progression-free survival of OC patients [18], we selected this miRNA as the target for the LINC00665. To further reveal the relationship between LINC00665 and miR181a-5p, we constructed the overexpress miR-181a-5p (Fig. 3B, p<0.01) and the interaction relation between the two above was confirmed by dual-luciferase reporter assay (Fig. 3C). Moreover, our results show that LINC00665 knockdown upregulated miR-181a-5p expression in SKOV-3 and OVCAR-3 (Fig. 3D). In addition, RT-qPCR proved that compared with ISOE80, the transcription of miR-181a-5p was significantly reduced in SKOV-3, A2780, OVCAR-3 and HO8910 cell lines (Fig. 3E).

\section{MiR-181a-5p regulates FHDC1 expression in SKOV-3 and OVCAR-3 cells}

As predicted by TargetScan (http://www.targetscan.org), FHDC1 was one of the target genes of miR181a-5p (Fig. 4A). Since FHDC1 was reported to affect the cell cycle and cell division which related to tumor cell proliferation [19], dual-luciferase reporter assay to verify this interaction and observed that miR181a-5p overexpression sharply downregulated FHDC1-WT reporter vector's luciferase activity (Fig. 4B, $p<0.01$ ). Meanwhile, overexpression miR-181a-5p with a mutation within the predicted miR-181a-5p binding site did not influence luciferase activity of the FHDC1 reporter vector (Fig. 4B). We determined 
both the RNA and protein expression level of FHDC1 overexpressed miR-181a-5p with RT-qPCR, western blot and immunofluorescence. The results demonstrated that FHDC1 was significantly downregulated in the SKOV-3 and OVCAR-3 cells overexpressed miR-181a-5p compared to negative control (Fig. 4C, 4D and 4E). Similarity, FHDC1 level was significantly up regulated in SKOV-3, A2780, OVCAR-3 and HO8910 as comparison to the ISOE80 (Fig. 4F).

\section{miR-181a-5p/FHDC1 mediated the effect of LINC00665 knockdown on SKOV-3 and OVCAR-3 cells}

To confirm the interaction of miR-181a-5p, FHDC1 and LINC00665 in OC, we co-transfected SKOV-3 cells with sh-LINC00665 alone, or sh-LINC00665 with miR-181a-5p inhibitor, or their combinations with shFHDC1. RT-qPCR results showed that after transfection of miR-181a-5p inhibitor, the expression of miR181a-5p in cells was significantly down-regulated. However, in sh-LINC00665 transfected cells, FHDC1 was significantly down-regulated (Figure $5 \mathrm{~A}, \mathrm{p}<0.01$ ). CCK8 assay revealed that the cells transfected shLINC00665 and NC inhibitor showed lowest cell viability, while the viability of cells transfected shLINC00665 along with miR-181a-5p inhibitor and sh-FHDC1 markedly increased compared to the cells transfected sh-LINC00665 and miR-181a-5p (Fig. 5B, p<0.05). Besides, as shown in Fig. 5C and Fig. 5D, the cells transfected sh-LINC00665 and NC inhibitor showed highest level of cell apoptosis. Downregulation of miR-181a-5p remarkably inhibited the cell apoptosis induced by sh-LINC00665. Meanwhile, sh-FHDC1 markedly reversed the inhibition effect of miR-181a-5p inhibitor on SKOV-3 cells apoptosis $(p<0.05)$. Furthermore, the wound healing assay delineated that downregulation of miR-181a$5 p$ remarkably improved the migration of the SKOV-3 cells with LINC00665 knockdown (Fig. 5E), compared to the cells co-transfected with sh-LINC00665 and NC inhibitor, while sh-FHDC1 markedly reversed the improvement from miR-181a-5p inhibitor (Fig. 5F, $p<0.05$ ). Similarly, the transwell assay delineated that the downregulation of miR-181a-5p markedly aggravated the invasion of SKOV-3 cells with LINC00665 knockdown. And sh-FHDC1 remarkably inhibited such a restoration effect (Fig. 5F, $p<0.05)$.

\section{Discussion}

Ovarian carcinoma presents its high frequency and aggressiveness among all the malignancies in women around the word. Exploring the molecular mechanisms, finding potential diagnostic biomarkers and therapeutic targets maybe the ideal ways to reduce the harm of ovarian cancer to women. In the present research, LINC00665 was up regulated. Once the LINC00665 is knockdown, the cell viability is dramatically reduced and the the cell cycle at the G0/G1 phase is arrested ,meanwhile the migration and invasion of SKOV-3 and OVCAR-3 cells is inhibited in vitro. This preliminary research indicated that LINC00665 might be a promising candidate in ovarian cancer therapy.

Increasing evidence indicates that dysregulation of IncRNAs affected tumorigenesis and metastasis in human cancer [20]. In ovarian cancer, several IncRNAs have been identified as indicators for patients' prognosis. For instance, the expression of IncRNA ROR has been studied evidently higher in the plasma of ovarian cancer patients than that in healthy controls [21]. In contrast, in ovarian cancer tissues, 
comparing with the patients whose expression of CASC15 expression is higher, the low expression of CASC15 present a better overall and progression-free survival [22]. In the study, we demonstrated that LINC00665 was remarkably higher in ovarian cancer cell lines (SKOV-3, A2780, OVCAR-3 and H08910). LINC00665 is first reported in oral premalignant lesions [11]. Next, LINC00665 is observed upregulated in several other tumors, including hepatocellular carcinoma [12] and lung adenocarcinoma [13]. For example, in gastric cancer, LINC00665, as the ceRNA of miR-149-3p, inhibits the viability and invasion of gastric cancer cells by regulating RNF2 [23]. In prostate cancer, LINC00665 functions as a sponge of miR1224-5p, and silencing LINC00665 can sharply reduce the growth and metastasis of PC cells [24]. In this study, we demonstrated that LINC00665 knockdown inhibited the proliferation, migration and invasion of OC cells (SKOV-3 and OVCAR-3).

Above evidence suggests that LINC00665 plays a role of miRNA sponges to downregulate the activity of target miRNAs in many cancers $[23,24]$. Herein, we identified miR-181a-5p as the target miRNAs of LINC00665, as they have complementary binding sites. And again, by the same method we predicted FHDC1 was the target of miR-181a-5p and they were validated with the luciferase assay. A previous study has shown that miR-181a-5p was downregulated in aggressive human breast and colon cancers, and indicated that miR-181a-5p inhibited tumor cell growth and invasion by attenuation elevated MMP-14 expression [25]. Most importantly, a 164 matched tumor biopsies study has shown that miR-181a-5p is related to overall and progression-free survival $(p<0.05)$, residual tumor volume and Pt-free interval in ovarian cancer [18].

Previous studies highlingt the important role of FHDC1 in maintenance of the Golgi-derived microtubule network. Endogenous FHDC1 has the association with the formation of Golgi ribbon. When the expression of FHDC1 is decreased, the defective Golgi assembly occurs[26]. Besides that, FHDC1 is found to affect the cell cycle and cell division [19]. We found that LINC00665 downregulated the expression of miR-181a-5p via direct interacting and upregulating the expression of FHDC1 in SKOV-3 and OVCAR-3 cells. At the same time, we found that miR-181a-5p decreased the expression of FHDC1 and reversed the effect of LINC00665 knockdown in SKOV-3 cells. Meanwhile, knockdown FHDC1 reversed the effect of miR-181a-5p inhibitor in SKOV-3 cells. All of these data indicated LINC00665 might take part in the inactivation of the miR-181a-5p-FHDC1 pathway.

In general, the present study proved that LINC00665 modulated miR-181a-5p/FHDC1 pathway to facilitate ovarian cancer progression. Our investigation suggested that LINC00665/miR-181a-5p/FHDC1 signal axis might provide a new therapeutic target for ovarian cancer therapy.

\section{Declarations}

\section{Ethics approval and consent to participate}

Not applicable.

\section{Consent for publication}


Not applicable.

\section{Availability of data and materials}

All data generated or analysed during this study are included in this published article.

\section{Competing interests}

The authors state that there are no conflicts of interest.

\section{Funding}

None.

\section{Authors' contributions}

S.W conducted most of the experiments. J.W. interpreted and analyzed the data. Y.W wrote the first draft of the article. J.L. finalized the manuscript. K.L. conceived the study and revised the manuscript. All authors read and approved the final manuscript.

\section{Acknowledgements}

Not applicable.

\section{References}

1. Bray F, et al. Global cancer statistics 2018: GLOBOCAN estimates of incidence and mortality worldwide for 36 cancers in 185 countries. CA Cancer J Clin. 2018;68(6):394-424.

2. Torre LA, et al. Ovarian cancer statistics, 2018. CA Cancer J Clin. 2018;68(4):284-96.

3. Zhang ML, et al. [Report of breast cancer incidence and mortality in China registry regions, 20082012]. Zhonghua Zhong Liu Za Zhi. 2019;41(4):315-20.

4. Marth C, Reimer D, Zeimet AG. Front-line therapy of advanced epithelial ovarian cancer: standard treatment. Ann Oncol. 2017;28 Suppl 8:viii36-9.

5. Qiu MT, et al. Long noncoding RNA: an emerging paradigm of cancer research. Tumour Biol. 2013;34(2):613-20.

6. Lalevee S, Feil R. Long noncoding RNAs in human disease: emerging mechanisms and therapeutic strategies. Epigenomics. 2015;7(6):877-9.

7. DiStefano JK. The Emerging Role of Long Noncoding RNAs in Human Disease. Methods Mol Biol. 2018;1706:91-110.

8. Xue M, Zhuo Y, Shan B. MicroRNAs, Long Noncoding RNAs, and Their Functions in Human Disease. Methods Mol Biol. 2017;1617:1-25. 
9. Sun X, et al., Long non-coding RNA LINC00968 reduces cell proliferation and migration and angiogenesis in breast cancer through up-regulation of PROX1 by reducing hsa-miR-423-5p. Cell Cycle, 2019. 18(16): p. 1908-1924.

10. Lv P, et al. Long non-coding RNA SNHG6 enhances cell proliferation, migration and invasion by regulating miR-26a-5p/MAPK6 in breast cancer. Biomed Pharmacother. 2019;110:294-301.

11. Jia H, Wang $X$, Sun Z. Exploring the molecular pathogenesis and biomarkers of high risk oral premalignant lesions on the basis of long noncoding RNA expression profiling by serial analysis of gene expression. Eur J Cancer Prev. 2018;27(4):370-8.

12. Wen DY, et al. Expression of the Long Intergenic Non-Protein Coding RNA 665 (LINC00665) Gene and the Cell Cycle in Hepatocellular Carcinoma Using The Cancer Genome Atlas, the Gene Expression Omnibus, and Quantitative Real-Time Polymerase Chain Reaction. Med Sci Monit. 2018;24:2786808.

13. Cong Z, et al. Long non-coding RNA linc00665 promotes lung adenocarcinoma progression and functions as ceRNA to regulate AKR1B10-ERK signaling by sponging miR-98. Cell Death Dis. 2019;10(2):84.

14. Adami GR, et al. Effects of green tea on miRNA and microbiome of oral epithelium. Sci Rep. 2018;8(1):5873.

15. Marshall J. Transwell((R)) invasion assays. Methods Mol Biol. 2011;769:97-110.

16. Ferracin $\mathrm{M}$, et al. Absolute quantification of cell-free microRNAs in cancer patients. Oncotarget. 2015;6(16):14545-55.

17. Katoh M, Katoh M. Identification and characterization of human FHDC1, mouse Fhdc1 and zebrafish fhdc1 genes in silico. Int J Mol Med. 2004;13(6):929-34.

18. Petrillo $M$, et al. Identification of high-grade serous ovarian cancer miRNA species associated with survival and drug response in patients receiving neoadjuvant chemotherapy: a retrospective longitudinal analysis using matched tumor biopsies. Ann Oncol. 2016;27(4):625-34.

19. Chen HQ, et al. Gene expression network regulated by DNA methylation and microRNA during microcystin-leucine arginine induced malignant transformation in human hepatocyte L02 cells. Toxicol Lett. 2018;289:42-53.

20. Dai L, et al. p53 Plays a Key Role in the Apoptosis of Human Ovarian Cancer Cells Induced by Adenovirus-Mediated CRM197. Hum Gene Ther. 2018;29(8):916-26.

21. Shen W, et al., Diagnostic Value of IncRNA ROR in Differentiating Ovarian Cancer Patients. Clin Lab, 2020. 66(7).

22. Shi Y, et al. LncRNA CASC15 Functions As An Unfavorable Predictor Of Ovarian Cancer Prognosis And Inhibits Tumor Progression Through Regulation Of miR-221/ARID1A Axis. Onco Targets Ther. 2019;12:8725-36.

23. Qi H, Xiao Z, Wang Y. Long non-coding RNA LINC00665 gastric cancer tumorigenesis by regulation miR-149-3p/RNF2 axis. Onco Targets Ther. 2019;12:6981-90. 
24. Chen W, et al. LncRNA LINC00665 Promotes Prostate Cancer Progression via miR-1224-5p/SND1 Axis. Onco Targets Ther. 2020;13:2527-35.

25. Li Y, et al. miR-181a-5p Inhibits Cancer Cell Migration and Angiogenesis via Downregulation of Matrix Metalloproteinase-14. Cancer Res. 2015;75(13):2674-85.

26. Copeland SJ, Thurston SF, Copeland JW. Actin- and microtubule-dependent regulation of Golgi morphology by FHDC1. Mol Biol Cell. 2016;27(2):260-76.

\section{Figures}
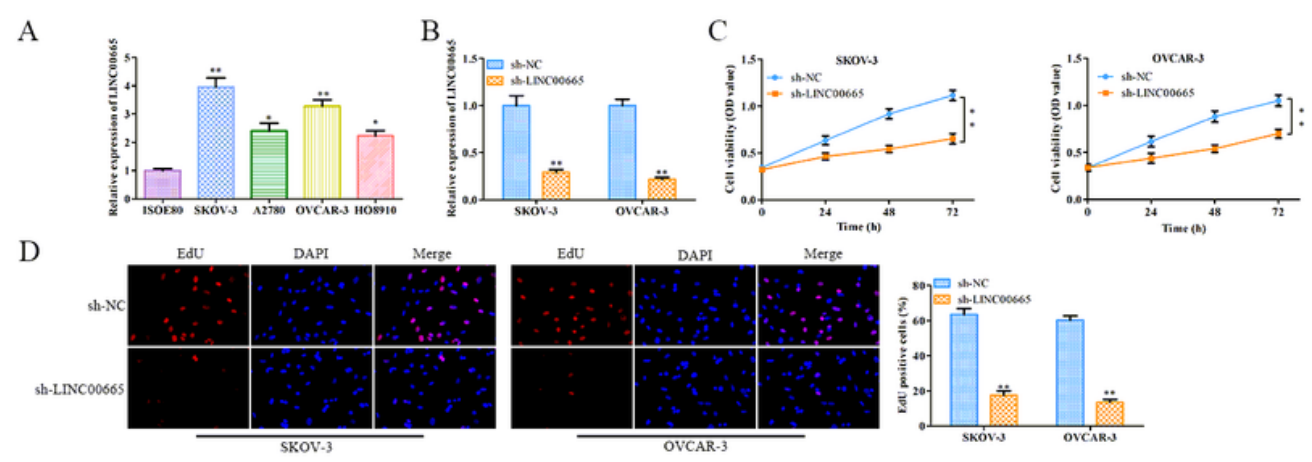

$\mathrm{E}$
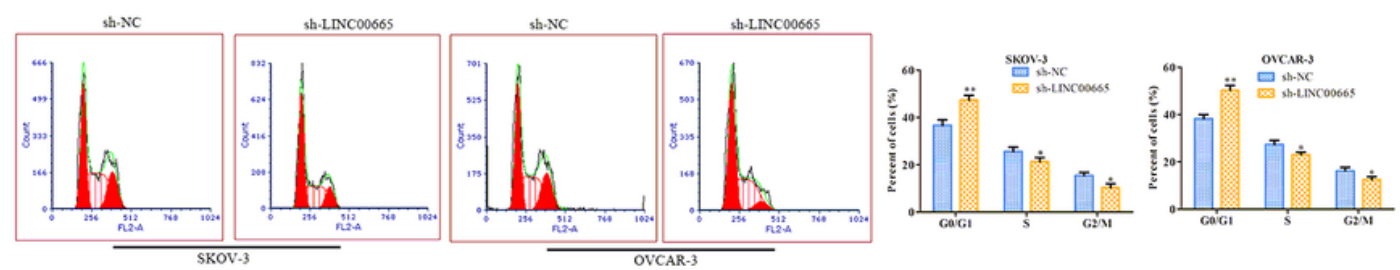

$\mathrm{F}$
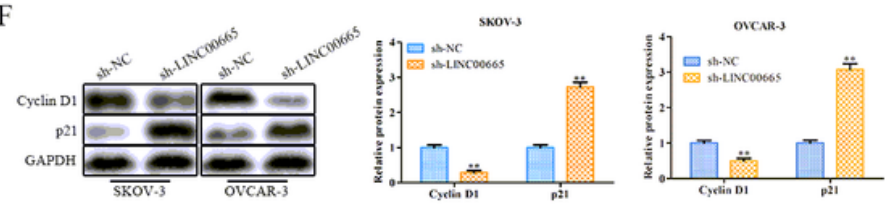

G
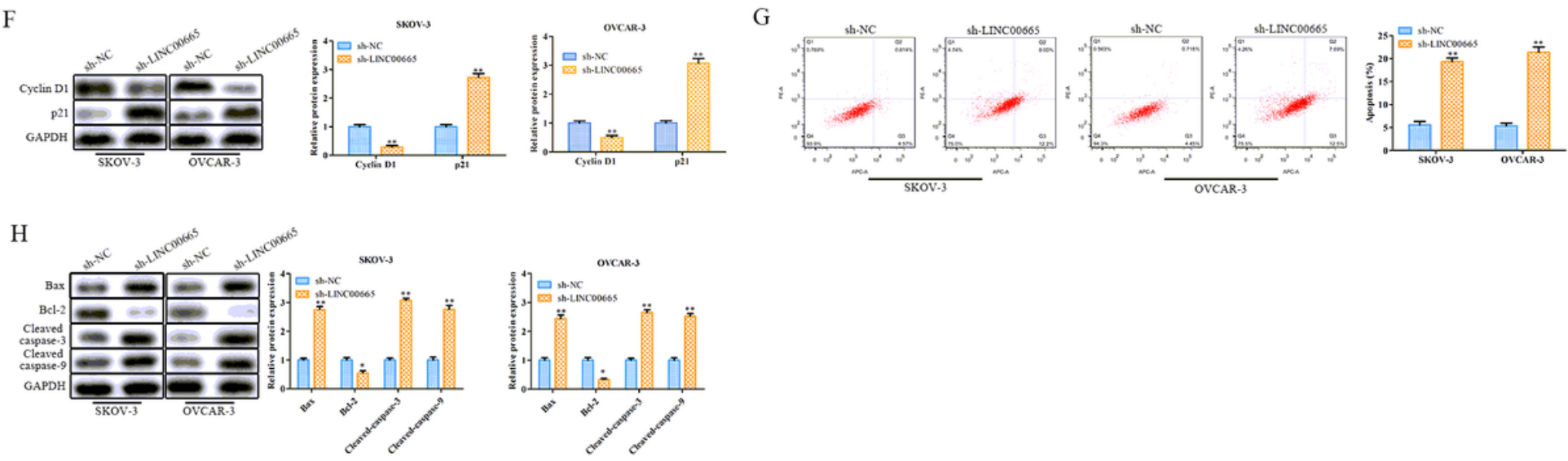

Figure 1

In order to take the further exploration about the important role of LINC00665 in ovarian cancer, we transfected sh-RNA into SKOV-3 and OVCAR-3 cells which targets to LINC00665 (sh-LINC00665). The expression of LINC00665 was detected by RT-qPCR and the result indicated that LINC00665 is significantly down-regulated. 
A
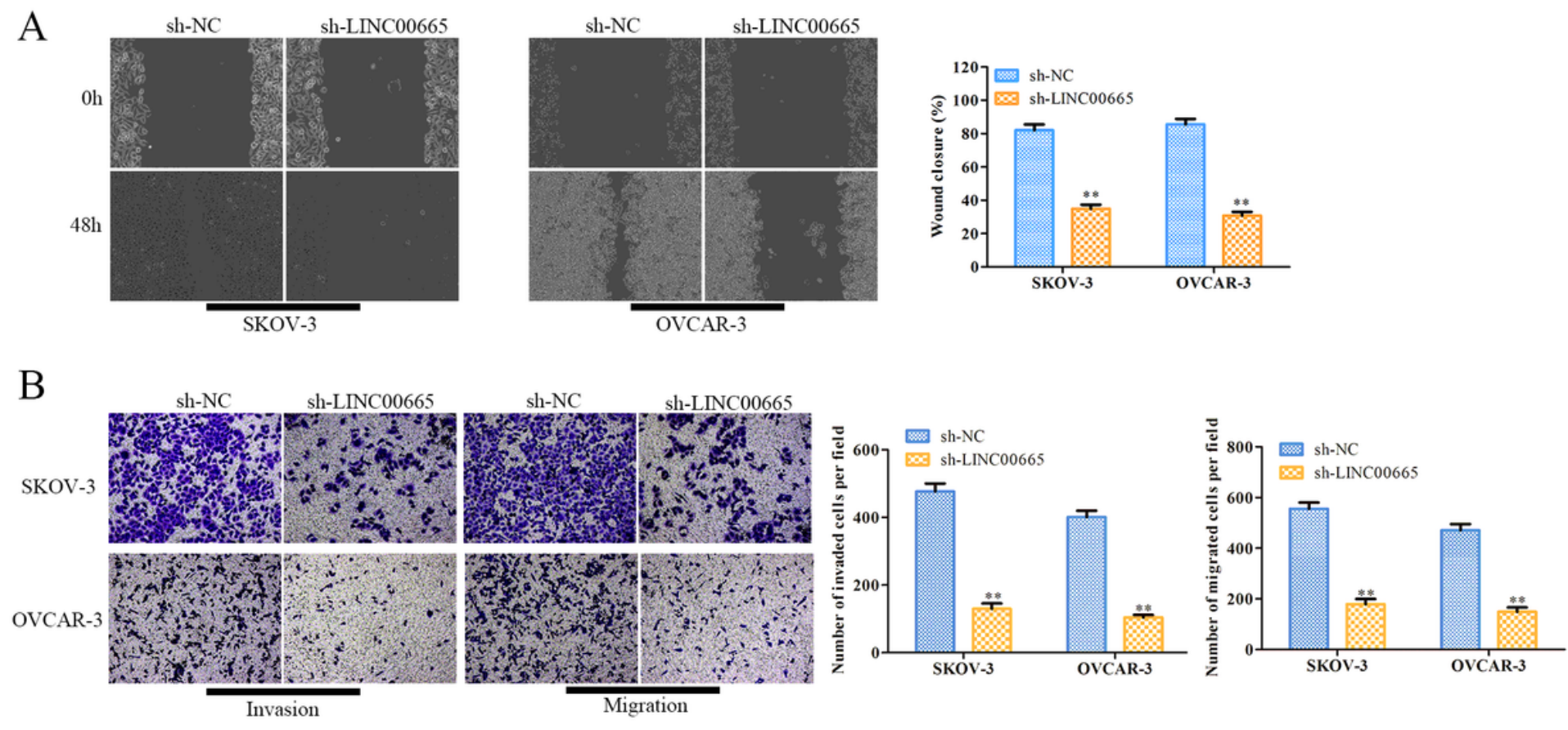

$\mathrm{C}$
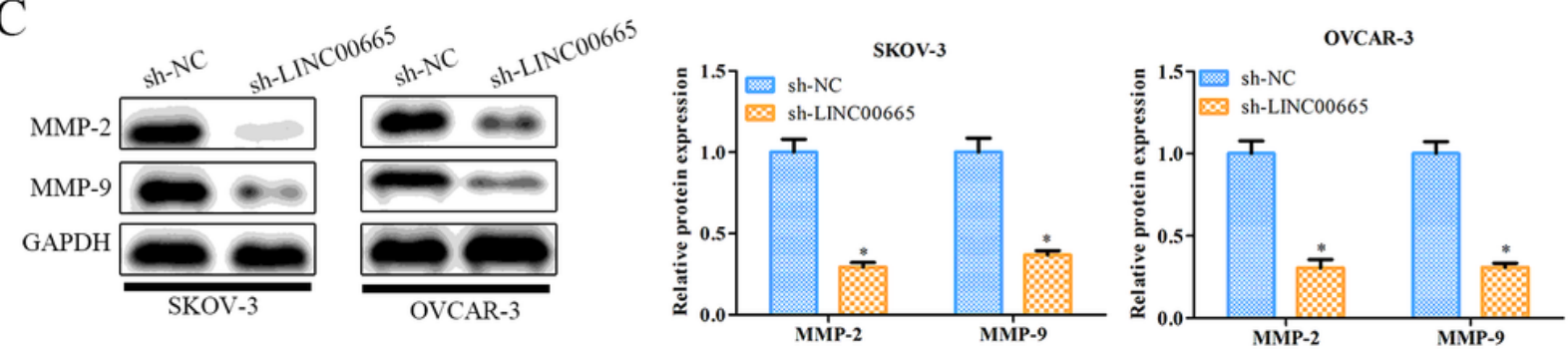

Figure 2

As presented in Fig. 2A, compared with the control group, the expression of LINC00665 reduced and the migratory ability of SKOV-3 and OVCAR-3 were remarkably repressed $(p<0.01)$. 


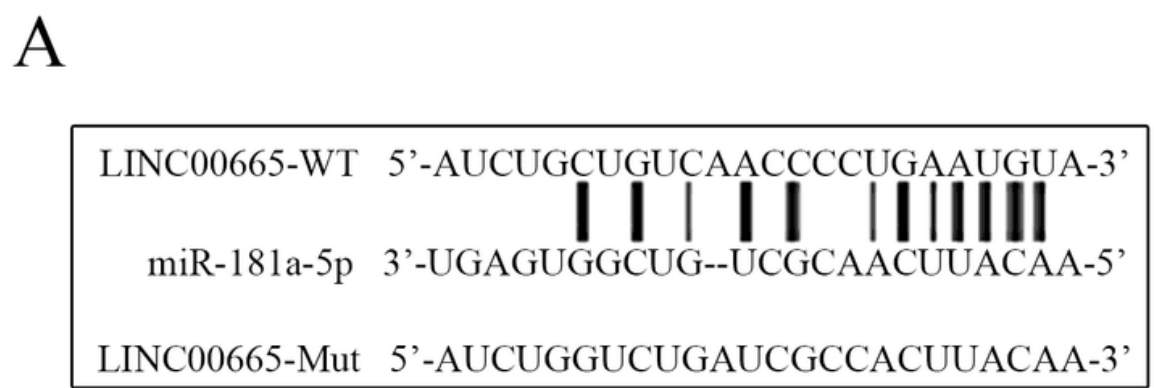

$\mathrm{C}$

SKOV-3

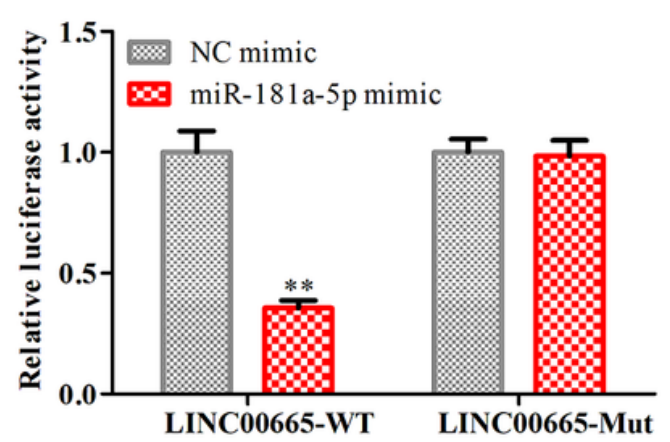

$\mathrm{D}$

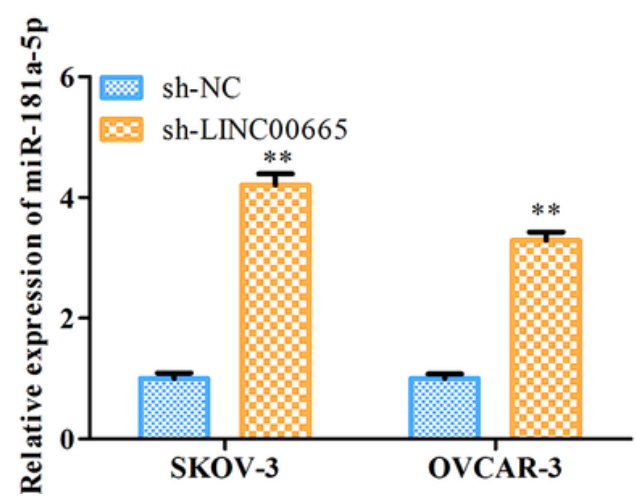

B

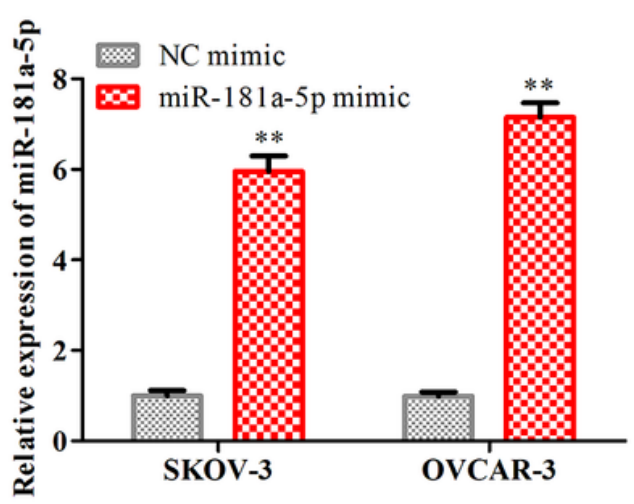

OVCAR-3

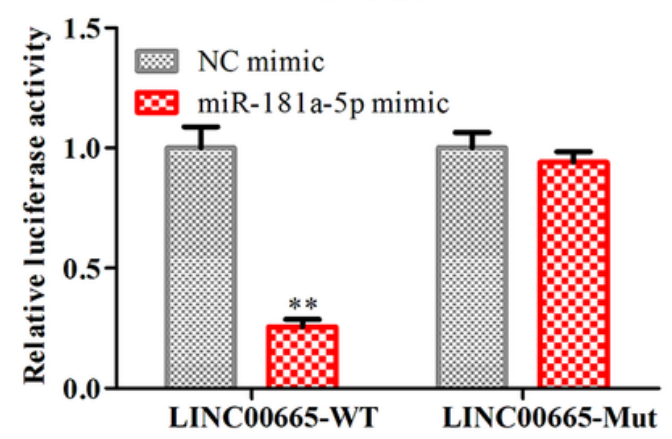

E

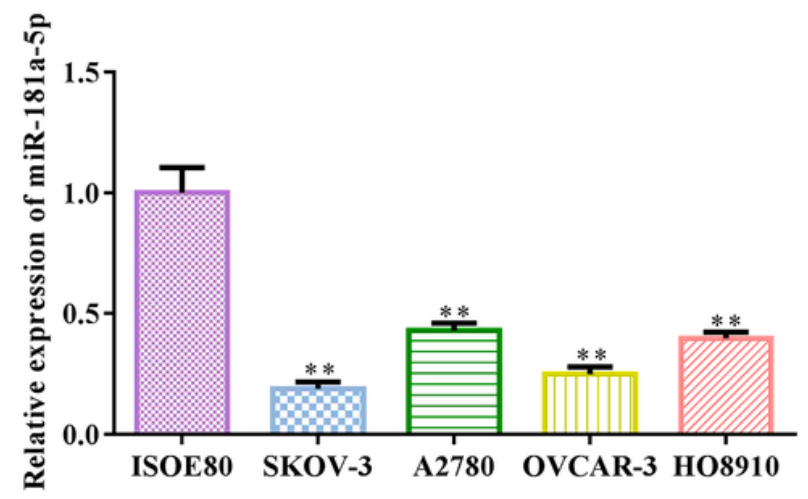

\section{Figure 3}

Since one way IncRNAs regulated gene expression was to act as a miRNA sponge, we predicted the potential miRNAs targets of LINC00665 in DIANA-LncBase (https://bigd.big.ac.cn). 
A

\begin{tabular}{|c|c}
\hline FHDC1 WT & 5 '-gaaauugcuuucagaaGAAUGUa-3' \\
Hsa-miR-181a-5p & 3 '-ugaguggcugucgcaaCUUACAa-5 ' \\
FHDC1 Mut & 5 '-gaaauugcuuucagaaCUUACAa-3'
\end{tabular}

$\mathrm{C}$

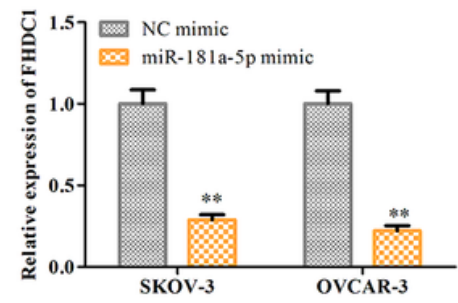

$\mathrm{E}$

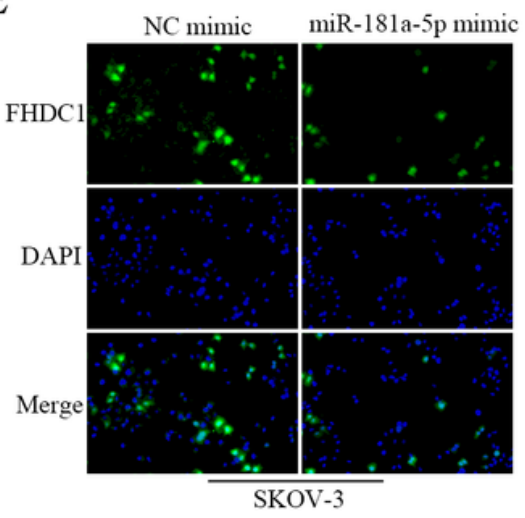

$\mathrm{D}$
B

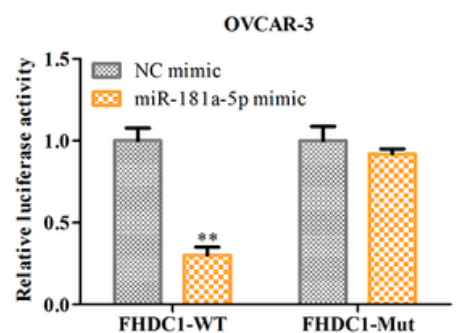

SKOV-3

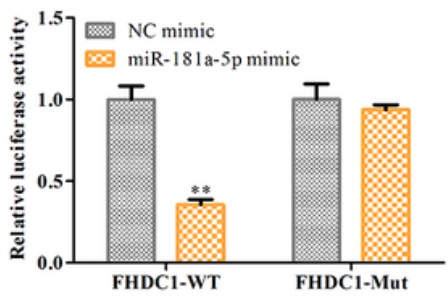

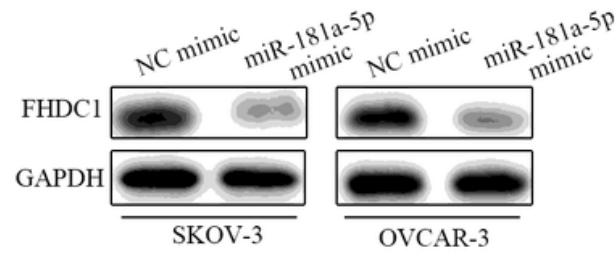

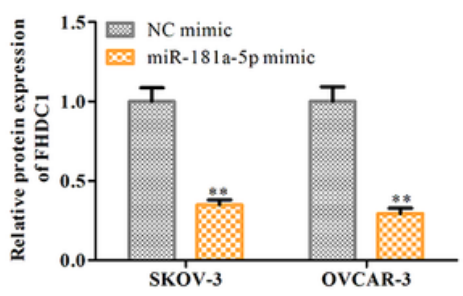

F

NC mimic miR-181a-5p mimic
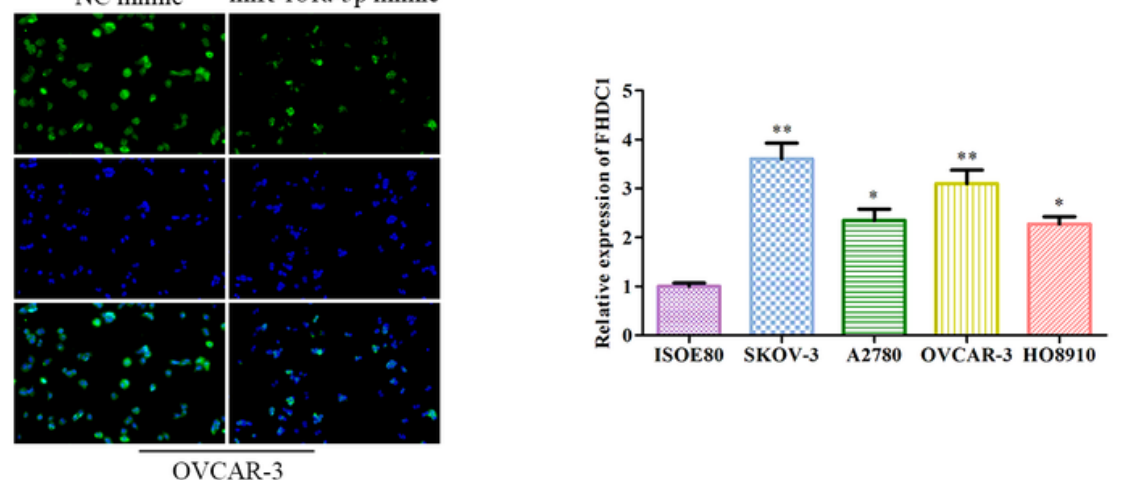

Figure 4

As predicted by TargetScan (http://www.targetscan.org), FHDC1 was one of the target genes of miR181a-5p. 
A

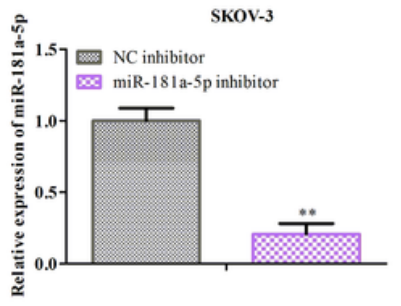

SKOV-3

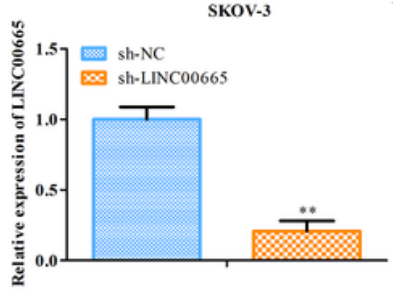

B

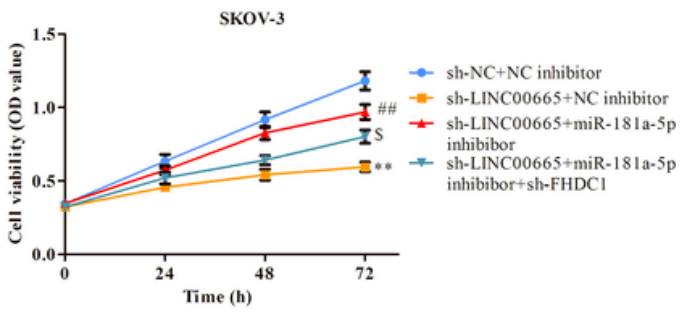

C
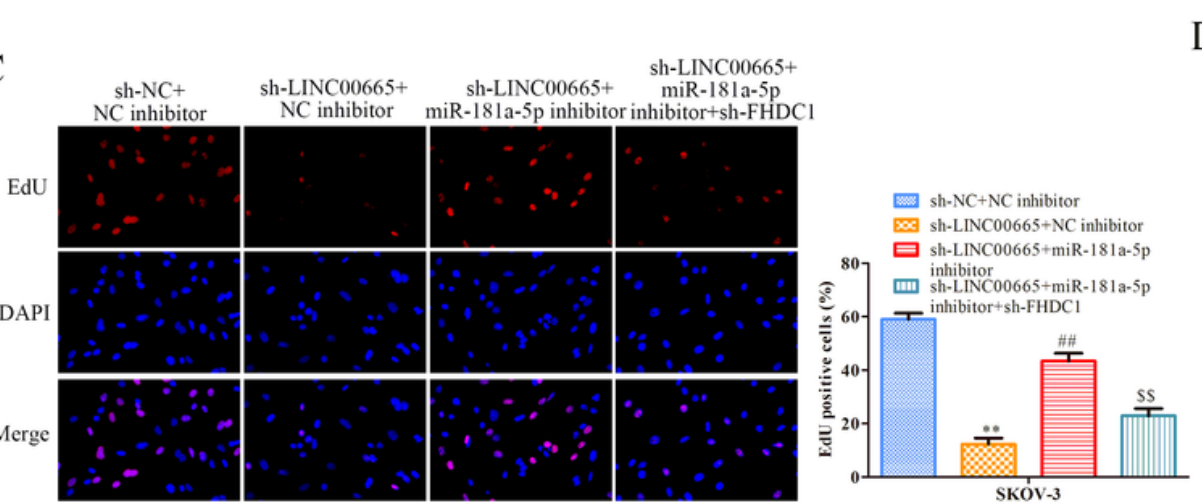

$\mathrm{E}$
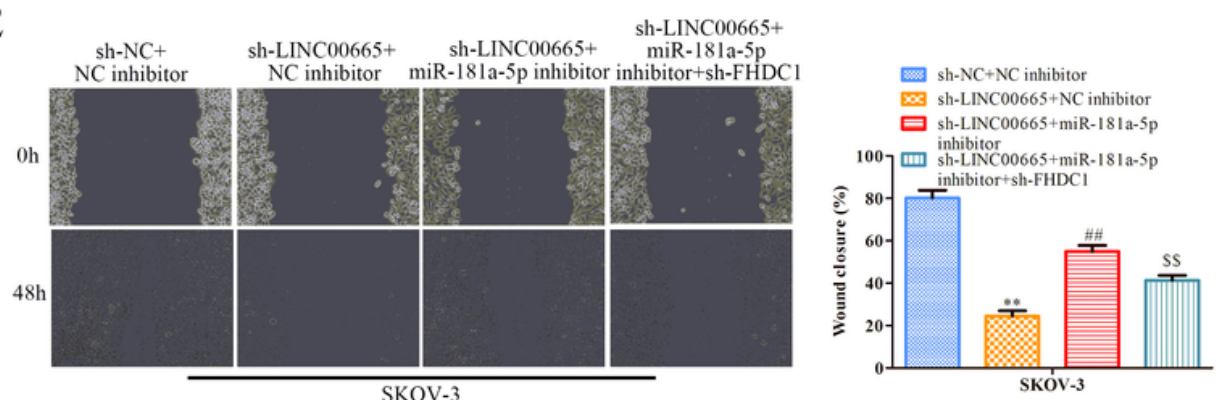

F
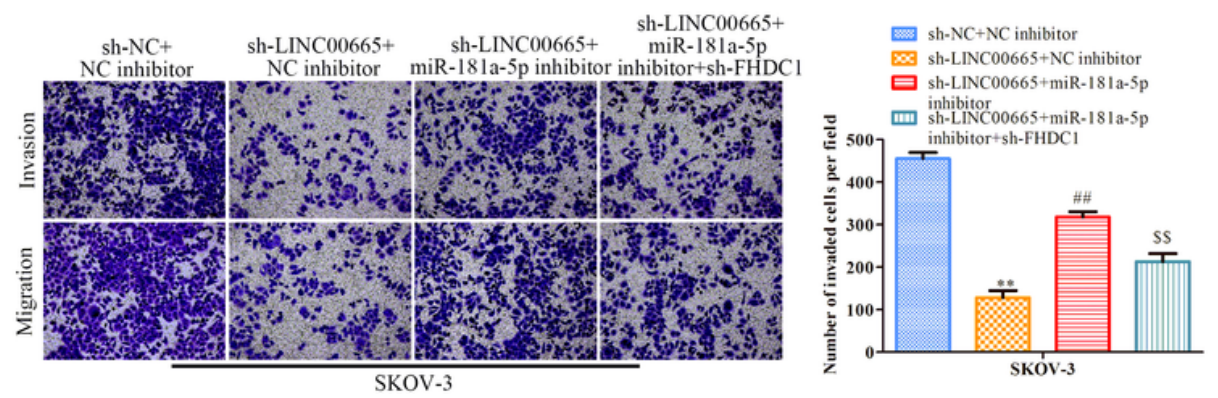

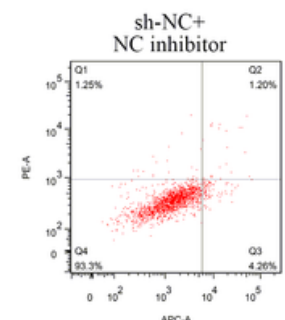

APCA

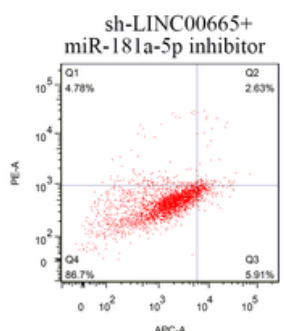

sh-NC+NC inhibitor

sh-LINC00665+NC inhibito

曰 sh-LINC00665+miR-181a-5p

四 $\begin{aligned} & \text { inhibitor } \\ & \text { sh-LINC00665+miR-181a-5p }\end{aligned}$

${ }^{25}$ inhibitor + sh-
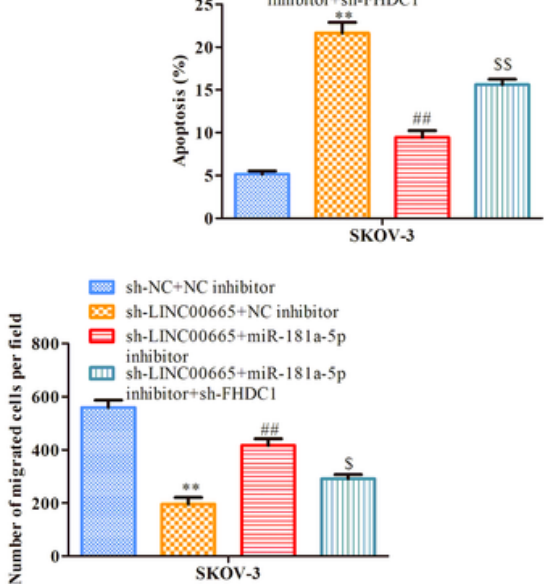

Figure 5

To confirm the interaction of miR-181a-5p, FHDC1 and LINC00665 in OC, we co-transfected SKOV-3 cells with sh-LINC00665 alone, or sh-LINC00665 with miR-181a-5p inhibitor, or their combinations with shFHDC1. RT-qPCR results showed that after transfection of miR-181a-5p inhibitor, the expression of miR181a-5p in cells was significantly down-regulated. However, in sh-LINC00665 transfected cells, FHDC1 was significantly down-regulated. 